\title{
Left Atrial Size After Cardioversion for Atrial Fibrillation: Effect of External Direct Current Shock
}

\author{
Anna Vittoria Mattioli, MD, Silvia Bonatti, MD, Lorenzo Bonetti, MD, and \\ Giorgio Mattioli, MD, Modena and Reggio Emilia, Italy
}

Objective. The aim of this study was to evaluate the effect of external direct current (DC) shock on left atrial (LA) dimension and volumes after cardioversion for atrial fibrillation, and the relation between $L A$ size and atrial function.

Methods. We evaluated 180 patients who were randomly cardioverted with DC shock (90 patients) or drugs (90 patients). Echocardiographic evaluations included LA size and volumes. LA passive and active emptying volumes were calculated, and LA function was measured as atrial ejection force. Changes in LA diameters and volumes were correlate with atrial systolic function.

Results. The LA was dilated in all patients during arrhythmia and decreased after the restoration of sinus rhythm. The entity of reduction was different in the $\mathbf{2}$ groups of patients. LA maximal and minimal volumes were increased after DC shock as compared

$\mathrm{T}$ he relationship between left atrial (LA) size and atrial fibrillation (AFib) is controversial. It is well known that AFib causes atrial dilation, and progressive LA enlargement occurs once AFib becomes chronic. ${ }^{1,2}$ Conversely, dilatation may partially regress if in sinus rhythm is restored. ${ }^{3}$ The Stroke Prevention in Atrial Fibrillation (SPAF) study showed that multiple factors contribute to LA enlargement, including the presence and persistence of the arrhythmia. ${ }^{4}$ The Framingham study showed a relation between LA size and the risk of stroke in men, and the risk of death in both sexes. 5,6 This study evaluates the changes in LA size and function after direct current (DC) shock for AFib.

From the Department of Cardiology, University of Modena and Reggio Emilia, Italy.

Reprint requests: Anna Vittoria Mattioli, MD, Department of Cardiology, University of Modena, Via del pozzo, 71, 41100 Modena, Italy (E-mail: mattioli.annavittoria@unimo.it).

Copyright 2003 by the American Society of Echocardiography.

0894-7317/2003/\$30.00+0

doi:10.1067/mje.2003.2 with patients treated with drugs (LA maximal volume $34 \pm 4$ vs $31 \pm 5 ; P<.01 ;$ LA minimal volume $18 \pm 2.6$ vs $15 \pm 3.6 ; P<.01)$. The atrial function was also depressed after DC shock and the delay in the recovery of atrial contractility was related to LA dilation. Patients treated with drugs had a higher atrial ejection force that was associated with a more marked reduction in LA maximal volume after the restoration of in sinus rhythm. A relationship between LA volumes and atrial ejection force was observed in the group of patients with depressed atrial mechanic function $(r=-0.78 ; P<.001)$. The active emptying fraction was lower, although not significantly, in this group, whereas the conduit volume was increased. Conclusion. External DC shock induced a depressed atrial mechanic function in many patients and this was associated with a persistence of LA dilation. (J Am Soc Echocardiogr 2003;16:271-6.)

\section{METHODS}

\section{Study Population}

Patients who were hemodynamically stable and referred for cardioversion for nonrheumatic AFib were considered for inclusion in this study. The study group included 255 consecutive patients with a primary diagnosis of AFib. To be included, patients needed documentation of the arrhythmia by electrocardiography. The onset of AFib was defined as "a new and clearly recognizable onset of symptoms including palpitations, dyspnea, dizziness, or a combination of such symptoms." Exclusion criteria were atrial flutter, recent acute myocardial infarction, reduced ejection fraction $<45 \%$, episodes of heart failure, mitral and aortic valvular stenosis and prosthesis, more than mild mitral and aortic insufficiency, atrial and/or left-ventricular thrombosis, spontaneous echocontrast, and spontaneous cardioversion of the arrhythmia. We also excluded patients who were admitted with another diagnosis and patients who had secondary AFib. Of the initial 255 patients, 75 were excluded because they did not meet the inclusion criteria. The remaining 180 patients randomly receive pharmacologic or electric cardioversion. Patients were classified as group 1 if they underwent external electric cardioversion $(n=90)$, and group 2 if they were treated with procainamide hydrochloride or propafenone hydrochloride $(\mathrm{n}=90)$. At the time of enrollment, 
patients were on the following medications for ventricular rate control: digoxin ( $n=39$ patients; 21.6\%) and propafenone hydrochloride ( $\mathrm{n}=12$ patients; $6.6 \%$ ). No patients received therapy with specific calcium antagonists. All patients were hospitalized because of an acute episode of AFib, they were treated with anticoagulants and underwent transesophageal echocardiography then cardioversion was performed. Patients with echocardiographic risk factors as indicated by SPAF study were excluded from this study. ${ }^{7}$ These patients were treated with anticoagulants for at least 30 days and underwent a different protocol of cardioversion according to AFib guidelines. ${ }^{8}$ The decision to undergo either electric or chemical cardioversion was left to the referring physician unaware of the protocol. Clinicians could excluded a patient from the study on the basis of echocardiography findings but could not change the modality of cardioversion. Procainamide hydrochloride and propafenone hydrochloride were randomly chosen following a blind list. The protocol was approved by the ethical committee of our university and all patients signed an informed consent form with detailed information on the protocol.

\section{Cardioversion}

DC shock was performed with the use of anterior paddles in patients who were fasting and received intravenous sedation with propofol. A total of 77 patients (85.5\%) cardioverted with a single monophasic electric shock; 2 shocks were required in the other patients. The total amount of electric energy used for cardioversion was $250 \pm 190 \mathrm{~J}$ per patient. Pharmacologic cardioversion was performed using bolus procainamide hydrochloride (56 patients) or propafenone hydrochloride (34 patients). Procainamide hydrochloride was infused at the dosage of $100 \mathrm{mg}$ intravenously administered every 5 minutes up to a maximum dose of $1 \mathrm{~g}$. Propafenone hydrochloride was given at a dose of $2 \mathrm{mg} / \mathrm{kg}$ body weight intravenously over 30 minutes. As soon as normal in sinus rhythm was restored electrocardiogram rhythm strips were obtained. All patients who were cardioverted were treated with propafenone hydrochloride after the restoration of in sinus rhythm.

\section{Echocardiography}

A complete M-mode and 2-dimensional color Doppler echocardiogram was performed on each patient using a commercial echocardiograph (Hewlett Packard, Andover, Mass) with a 2.5-MHz probe. A transesophageal study was also performed before cardioversion in all patients to investigate the presence of LA thrombi and LA spontaneous echocontrast. The following parameters were measured and compared:

- LA size was measured during systole along the parasternal long-axis view from 2-dimensionally guided M-mode tracings. ${ }^{9}$

- LA volumes were determined at mitral valve opening (maximal volume), at onset of atrial systole ( $\mathrm{P}$ wave of electrocardiogram, $\mathrm{P}$ volume), and at mitral valve clo- sure (minimal volume). LA volumes were measured from the apical 4- and 2-chamber views by means of the biplane area-length method and corrected for body surface area. ${ }^{10}$ From the LA volumes the following parameters were estimated. LA passive emptying was assessed as: LA passive emptying volume (maximal volume - $\mathrm{P}$ volume), conduit volume (left-ventricular stroke volume - [maximal volume - minimal volume]), and LA passive emptying fraction (LA passive emptying volume/maximal volume). LA active emptying was estimated using: LA active emptying volume (P volume - minimal volume) and LA active emptying fraction (LA active emptying volume/P volume). LA total emptying volume was also calculated: (maximal volume - minimal volume). Contribution of passive emptying volume ([maximal volume $-\mathrm{P}$ volume]/ stroke volume); conduit volume (conduit volume/ stroke volume); and active emptying volume ([P volume - minimal volume])/stroke volume) to left-ventricular stroke volume was also evaluated. ${ }^{11}$

- Mitral orifice area was assumed to be circular and estimated from the mitral annulus diameter as measured from the apical 4-chamber view.

- Transmitral pulsed Doppler was recorded from apical 4-chamber view, with the sample volume positioned between the tips of the mitral leaflets. Peak early filling (E) and atrial filling (A) velocities were recorded. Deceleration time and pressure half time were measured from the transmitral flow pattern.

- The atrial ejection force (AEF) (dynes) was evaluated using the following equation ${ }^{12}$ :

$$
0.5 \times \rho \times \text { mitral orifice area } \times(\text { peak A velocity })^{2} .
$$

The unit force was measured in dynes. The variability between measurements was previously assessed in our laboratory. ${ }^{13}$ Effective mechanic atrial function was defined as "the presence of AEF $>7$ dynes and of atrial filling wave peak velocity $>0.5 \mathrm{~m} / \mathrm{s}$."12,13 All measurements were taken during quiet breathing with patients in the left lateral decubitus position. All Doppler results are the mean measurements of 5 cardiac cycles. The Doppler signal was analyzed using an IBM computer (Milan, Italy). Thirty percent of beats measured for each patient were checked for accuracy by a second investigator (A.V.M.); $90 \%$ correlation was found between the analysts. A complete Doppler echocardiographic examination was performed immediately ( 1 hour), at day 1 , and day 7 after the restoration of in sinus rhythm. The follow-up was discontinued if the patients reverted to AFib (8 patients after 7 days and 9 after 15 days).

\section{Statistical Analysis}

Data are expressed as mean value \pm 1 SD for continuous variables. The statistical significance of serial changes in echocardiographic parameters was determined by analysis of variance for repeated measures. Values for patients of different groups were compared using the Student unpaired $t$ test. Because the mean age was not different 
Table 1 Demographic, clinical, and echocardiographic characteristics of patients

\begin{tabular}{lcc}
\hline & $\begin{array}{c}\text { Group 1 } \\
(\mathbf{n}=\mathbf{9 0})\end{array}$ & $\begin{array}{c}\text { Group 2 } \\
(\mathbf{n}=\mathbf{9 0})\end{array}$ \\
\hline Age (ys) & $60 \pm 16$ & $61 \pm 16$ \\
Male/female & $67 / 23$ & $61 / 29$ \\
Height $(\mathrm{cm})$ & $169 \pm 13$ & $171 \pm 9$ \\
Weight $(\mathrm{kg})$ & $84 \pm 20$ & $86 \pm 20$ \\
Body mass index $\left(\mathrm{kg} / \mathrm{m}^{2}\right)$ & $27 \pm 3$ & $26 \pm 2$ \\
Mean ventricular rate $(\mathrm{bpm})$ & $94 \pm 24$ & $95 \pm 26$ \\
Systemic hypertension No. patients $(\%)$ & $14(15)$ & $15(16)$ \\
Diabetes mellitus No. patients $(\%)$ & $11(12)$ & $10(11)$ \\
Coronary artery disease No. patients $(\%)$ & $4(4)$ & $3(3)$ \\
Left ventricular EF $(\%)$ & $51 \pm 10$ & $52 \pm 9$ \\
Systolic blood pressure (mm Hg) & $134 \pm 22$ & $132 \pm 12$ \\
Diastolic blood pressure (mm Hg) & $90 \pm 10$ & $89 \pm 11$ \\
\hline
\end{tabular}

$E F$, Ejection fraction.

among the groups compared, statistical adjustment was considered unnecessary. Pearson correlation coefficient was used to study the correlation between 2 parameters. A $P$ value $<.05$ was considered significant.

\section{RESULTS}

The mean duration of AFib in the total population was $64 \pm 23$ hours. Elective cardioversion for AFib was accomplished with DC shock in 87 patients (96.6\%; group 1) and pharmacologically in 51 patients (56.6\%; group 2). The mean duration of arrhythmia was $40 \pm 17$ hours in group 1 and $44 \pm$ 21 hours in group 2 ( $P=$ not significant). The 2 groups were similar with respect to age and underline cardiac diseases (Table 1). Baseline echocardiography showed that LA diameters and volumes were enlarged in all patients during AFib (Tables 2 and 3). Examinations performed 1 hour after the restoration of sinus rhythm showed a decrease of LA diameters and volumes in $36(70.5 \%)$ patients reverted with drugs, and in 47 (54\%) patients cardioverted with DC shock. Serial evaluation of LA diameters and volumes are shown in Tables 2 and 3. The reduction of anteroposterior diameter was more marked than the reduction of superoinferior diameter $(-7 \%$ vs $-10 \%$ in group 1 and $-13.1 \%$ vs $-17.9 \%$ in group 2). Comparing the 2 groups the reduction was more evident in group 2 compared with group 1 (Figure 1). Atrial mechanic function was measured using peak A wave velocity and AEF. The initial values of the peak $A$ wave velocity and of AEF in patients who underwent DC shock were significantly lower compared with patients in group 2 (peak A wave $38 \pm 16$ vs $45 \pm 20 ; P<.001$ and AEF $6 \pm 1.6$ vs $9 \pm 2 ; P<.001)$.

Effective atrial mechanic function was considered if AEF was found to be $>7$ dynes; in group $1, \mathrm{AEF}$ was $>7$ dynes in 46 patients $(52.8 \%)$ compared with
41 patients in group $2(80.3 \% ; P<.01)$. An AEF $>7$ dynes was associated with a more marked reduction in LA size after the restoration of in sinus rhythm. LA volumes were greater in patients who showed a delay in the restoration of atrial mechanic function and an AEF $<7$ dynes. This group included a higher number of patients who underwent DC shock. The active emptying volume was lower, although not significantly, in patients with $\mathrm{AEF}<7$ dynes group ( $5 \pm 2.6$ vs $7 \pm 2.3 ; P=.03$ ) whereas the passive emptying volume was similar to patients with an AEF $>7$ dynes $(10 \pm 2.9$ vs $9 \pm 3 ; P=$ not significant). The conduit volume was increased in patients who had an AEF $<7$ dynes compared with patients with a normal AEF $(30 \pm 5.9$ vs $28 \pm 3.6$ $P<.05)$. At day 7 we reported 5 recurrences of AFib in patients with a reduced atrial function ( 3 patients in group 1, and 2 patients in group 2) and 3 recurrences in patients with a normal atrial mechanic function (1 patient in group 1 , and 2 patients in group 2). At day 7, 44 patients (93\%) who underwent successful pharmacologic cardioversion showed a good recovery of atrial mechanic function whereas in the group of patients who underwent successful DC shock, 74 patients (98\%) showed a good atrial mechanic function.

Table 4 shows the correlation between LA volumes and diameters and AEF. We found a good inverse correlation between AEF and LA diameters in patients with a depressed atrial function (AEF $<7$ dynes), and the correlation was stronger for the superoinferior diameter $(r=-0.76 ; P<.001)$ (Figure 2). LA maximal volume after cardioversion was also inversely correlated with AEF $(r=-0.79$; $P<.001)$.

\section{DISCUSSION}

This study evaluated the effect of DC shock on LA volumes and diameter and the relationship between anatomic changes and atrial mechanic function. The salient observation from this study is that both LA size reduction and function are significantly depressed after electric cardioversion. A second observation was that the duration of AFib was shorter in patients who accomplished successful cardioversion independently on the modality. LA diameters and volumes were measured before and after cardioversion and were found to be reduced after the recovery of in sinus rhythm. Nevertheless, we reported a different rate of reduction among different patients. The modality of cardioversion seems to influence the magnitude of reduction. The reduction of atrial size was more marked in patients after chemical compared with electric cardioversion. We observed that the different modality of cardioversion can also affect the recovery of atrial mechanic function. 
Table 2 Changes in left-atrial diameters and volume after electric cardioversion of atrial fibrillation

\begin{tabular}{lcccc}
\hline & AFib & SR after CV & SR after 1 day & SR after 7 days \\
\hline LV end-diastolic diameter $(\mathrm{mm})$ & $56 \pm 3$ & $54 \pm 4$ & $53 \pm 3^{*}$ & $52 \pm 2.8^{*}$ \\
LV end-systolic diameter $(\mathrm{mm})$ & $36 \pm 3$ & $34 \pm 3.2$ & $33 \pm 3.1^{*}$ & $33 \pm 2.9^{*}$ \\
LV end-diastolic volume $\left(\mathrm{cm}^{3}\right)$ & $124 \pm 6$ & $123 \pm 8$ & $122 \pm 5$ & $123 \pm 7$ \\
LV end-systolic volume $\left(\mathrm{cm}^{3}\right)$ & $66 \pm 4$ & $65 \pm 7$ & $65 \pm 5$ & $65 \pm 6$ \\
LA size anteroposterior diameter $(\mathrm{mm})$ & $44 \pm 3$ & $41 \pm 3^{*}$ & $40 \pm 2.8 \dagger$ & $38 \pm 3 \dagger$ \\
LA size superoinferior diameter $(\mathrm{mm})$ & $48 \pm 2.9$ & $44 \pm 4^{*}$ & $43 \pm 5 \dagger$ & $41 \pm 4 \dagger$ \\
Maximal volume $\left(\mathrm{cm}^{3}\right)$ & $36 \pm 6$ & $34 \pm 4^{*}$ & $33 \pm 6^{*}$ & $32 \pm 5 \dagger$ \\
Minimal volume $\left(\mathrm{cm}^{3}\right)$ & $19 \pm 3$ & $18 \pm 2.6$ & $6 \pm 2.9^{*}$ & $14 \pm 2 \dagger$ \\
Atrial ejection force $($ dynes $)$ & $/ /$ & $9 \pm 2^{*}$ & $12 \pm 3.1 \dagger$ \\
\hline
\end{tabular}

$A F i b$, Atrial fibrillation; $S R$, In sinus rhythm; $L V$, left-ventricular; $L A$, left-atrial; $C V$, cardioversion.

${ }^{*} P<.01$ versus $A F i b ; \dagger P<.001$ versus AFib

Table 3 Changes in left-atrial diameters and volume after pharmacologic cardioversion of atrial fibrillation

\begin{tabular}{|c|c|c|c|c|}
\hline & AFib & SR after $\mathbf{C V}$ & SR after 1 day & SR after 7 days \\
\hline LV end-diastolic diameter ( $\mathrm{mm})$ & $55 \pm 4$ & $54 \pm 3.8$ & $52 \pm 3.1^{*}$ & $52 \pm 2.8^{*}$ \\
\hline LV end-systolic diameter (mm) & $36 \pm 4$ & $34 \pm 3$ & $33 \pm 3.2^{*}$ & $33 \pm 3^{*}$ \\
\hline LV end-diastolic volume $\left(\mathrm{cm}^{3}\right)$ & $123 \pm 5.6$ & $123 \pm 6.2$ & $122 \pm 5.7$ & $123 \pm 6$ \\
\hline LV end-systolic volume $\left(\mathrm{cm}^{3}\right)$ & $67 \pm 5$ & $67 \pm 6.7$ & $65 \pm 6$ & $65 \pm 6$ \\
\hline LA size anteroposterior diameter $(\mathrm{mm})$ & $43 \pm 2.9$ & $40 \pm 3.1^{*}$ & $38 \pm 2.6 \dagger$ & $38 \pm 3 \dagger$ \\
\hline LA size superoinferior diameter $(\mathrm{mm})$ & $46 \pm 3$ & $42 \pm 4^{*}$ & $40 \pm 5 \dagger$ & $39 \pm 4.3 \dagger$ \\
\hline Maximal volume $\left(\mathrm{cm}^{3}\right)$ & $36 \pm 6$ & $31 \pm 5 \dagger$ & $30 \pm 6 \dagger$ & $28 \pm 6 \dagger$ \\
\hline Minimal volume $\left(\mathrm{cm}^{3}\right)$ & $19 \pm 4$ & $15 \pm 3.6^{*}$ & $12 \pm 2.9 \dagger$ & $11 \pm 2.2 \dagger$ \\
\hline Atrial ejection force (dynes) & // & $9.2 \pm 1.6$ & $12 \pm 2^{*}$ & $13 \pm 2.8^{*}$ \\
\hline
\end{tabular}

$A F i b$, Atrial fibrillation; $S R$, In sinus rhythm; $C V$, cardioversion; $L V$, left-ventricular; $L A$, left-atrial.

${ }^{*} P<.01$ versus $A F i b ; \dagger P<.001$ versus AFib.

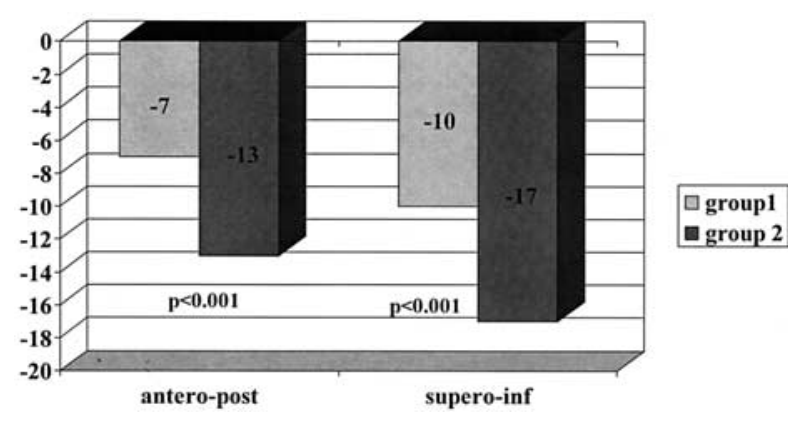

Figure 1 Reduction of left-atrial anteroposterior (anteropost) and superoinferior (supero-inf) diameters after cardioversion.

Previous reports showed that LA mechanic function is impaired after cardioversion of AFib, and the recovery of atrial function is delayed in patients who undergo DC shock compared with patients who undergo pharmacologic cardioversion. ${ }^{13,14}$ This study showed a correlation between recovery of atrial function and the size of the LA. The temporal course of return of atrial contraction velocities to normal values is associated with a reduction of LA volumes. The progressive augmentation of AEF, which occurs during successive intervals, was accompanied by a corresponding change in LA volumes in both group of patients. Patients who under-
Table 4 Correlation between echocardiographic parameters and atrial ejection force

\begin{tabular}{|c|c|c|c|c|}
\hline \multirow[b]{2}{*}{ Parameters } & \multicolumn{2}{|c|}{$\begin{array}{l}\text { Reduced atrial } \\
\text { ejection force } \\
<7 \text { dynes }\end{array}$} & \multicolumn{2}{|c|}{$\begin{array}{c}\text { Normal atrial } \\
\text { ejection force } \\
>7 \text { dynes }\end{array}$} \\
\hline & $r$ & $\boldsymbol{P}$ & $r$ & $\boldsymbol{P}$ \\
\hline $\begin{array}{l}\text { LA anteroposterior } \\
\text { diameter }\end{array}$ & $r=-0.51$ & .05 & $r=-0.26$ & .18 \\
\hline $\begin{array}{l}\text { LA superoinferior } \\
\text { diameter }\end{array}$ & $r=-0.76$ & .001 & $r=-0.24$ & .23 \\
\hline LA maximal volume & $r=-0.79$ & .001 & $r=-0.44$ & .03 \\
\hline LA minimal volume & $r=-0.48$ & .05 & $r=-0.43$ & .028 \\
\hline
\end{tabular}

$L A$, left-atrial.

went DC shock had a slower recovery of atrial function and of LA volumes reduction, implying a greater degree of atrial dysfunction. These findings are in agreement with previous studies. ${ }^{13,15}$

\section{LA Dilation and AFib}

It is controversial as to whether the LA enlargement in AFib is the result rather than the cause of AFib. Some investigators showed that AFib contributes to the enlargement of the atria. ${ }^{2,16}$ The SPAF study on LA enlargement demonstrated the relationship be- 
tween atrial size and AFib. The SPAF study showed that chronic AFib, long-lasting AFib, and systemic hypertension were independently associated with a large LA diameter. ${ }^{4} \mathrm{~A}$ recent study from the Mayo Clinic showed a relationship between LA volume and outcome event of AFib. LA volume was a significant and independent predictor of incident AFib in older patients. ${ }^{17}$ We reported a shorter duration of AFib in patients who were successfully cardioverted. This is the reason why our patients had just a mild dilation of LA. Nevertheless, this study suggests a direct relationship between the delay in the restoration of mechanic atrial function and a persistently dilated LA. This phenomenon is more evident after electric cardioversion and might be related to atrial electric remodeling. In diastole, the LA acts as a conduit between the pulmonary veins and the left ventricle, so that LA function is primarily influenced by the same factors that determine left-ventricular filling. ${ }^{18}$ The LA size is closely related to diastolic filling pressure. ${ }^{17,19}$ An increase in left-ventricular end-diastolic pressure causes LA stretch, which may contribute to the development of AFib. After cardioversion the LA remain dilated and vulnerable to AFib triggers. DC shock induced a delay in the recovery of atrial contraction. Anatomic changes occurring in the atria during AFib may have a deleterious effect persistent once sinus rhythm is restored. Biochemic and histologic changes in LA myocytes may be associated with anatomic changes in atrial volumes. ${ }^{20,21}$ We observed that smaller increments of LA diameter measured by M-mode echocardiography are associated with greater changes of LA volume. After cardioversion of AFib, the conduit function was enhanced in patients with a depressed atrial contractility whereas the contribution of the active emptying volume to left-ventricular stroke volume did not vary. The conduit volume was increased as a result of the increase in early diastolic filling. LA mechanic function is influenced by LA preload and afterload. ${ }^{22}$ No changes in atrial preload and afterload were observed in patients after DC shock. We can conclude that the decrease in atrial myocardial contractility may be the cause of the decrease in atrial performance. This depressed atrial contractility could be related to stunning induced by atrial electric remodeling. ${ }^{23}$

\section{Limitations}

The major limitation was that this study was not randomized. The decision between electric and chemical conversion was left to the referring physician. The other limitation was that, as in the usual clinical study, there is no baseline assessment of LA size and function in sinus rhythm before the onset of AFib.
LA max vol

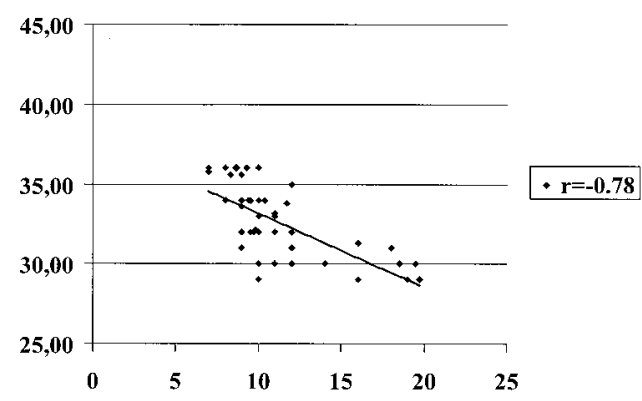

AEF

Figure 2 Correlation between left-atrial $(L A)$ volume ( $v o l)$ and atrial ejection force $(A E F)$. Max, maximal.

\section{Clinical Implications}

DC shock is the most successful approach of cardioversion but is associated with a prolonged atrial stunning. The duration of atrial stunning is variable depending on the duration of preceding AFib and atrial size. Shorter duration of arrhythmia and small LA dimension are associated with a less severe stunning. We report a more prolonged atrial stunning associated with electric cardioversion. This observation suggests that anticoagulant therapy after conversion of AFib is mandatory in patients who have undergone DC shock as a result of the increased embolic risk. Atrial stunning is related to dilation of atrial chambers. A persistent dilated LA could be the morphologic change associated with a reduced atrial systolic function.

\section{REFERENCES}

1. Sanfilippo AJ, Abascal VM, Sheehan M, Oertel LB, Harrigan P, Hughes RA, et al. Atrial enlargement as a consequence of atrial fibrillation: a prospective echocardiographic study. Circulation 1990;82:792-7.

2. Keren G, Etzion T, Sherez J, Zelcer AA, Megidish R, Miller $\mathrm{HI}$, et al. Atrial fibrillation and atrial enlargement in patients with mitral stenosis. Am Heart J 1987;114:1146-55.

3. Welikovitch L, Lafreniere G, Burggraf GW, Sanfilippo AJ. Change in atrial volume following restoration of sinus rhythm in patients with atrial fibrillation: a prospective echocardiographic study. Can J Cardiol 1994;10:993-6.

4. Dittrich HC, Pearce LA, Asinger RW, McBride R, Webel R, Zabalgoitia M, et al on behalf of the Stroke Prevention in Atrial Fibrillation Investigators. Left atrial diameter in nonvalvular atrial fibrillation: an echocardiographic study. Am Heart J 1999; 137:494-9.

5. Wolf PA, Abbott RD, Kannel WB. Atrial fibrillation as an independent risk factor for stroke: the Framingham study. Stroke 1991;22:983-8.

6. Benjamin EJ, D'Agostino RB, Belanger AJ, Wolf PA, Levy D. Left atrial size and the risk of stroke and death: the Framingham heart study. Circulation 1995;92:835-41.

7. The Stroke Prevention in Atrial Fibrillation Investigators. Predictors of thromboembolism in atrial fibrillation: echocar- 
diographic features of patients at risk. Ann Intern Med 1992; 116:6-12.

8. Guidelines for the management of patients with atrial fibrillation: a report of the American college of cardiology/American heart association task force on practice guidelines and the European society of cardiology committee for practice guidelines and policy conferences (committee to develop guidelines for the management of patients with atrial fibrillation) developed in collaboration with the North American society of pacing and electrophysiology. Eur Heart J 2001;22:1852-923.

9. Sahn DJ, DeMaria A, Kisslo J, Weiman A. Recommendations regarding quantitation in M-mode echocardiography: results of a survey of echocardiographic measurements. Circulation 1978;58:1072-83.

10. Ren JF, Kotler MN, De Pace NL, Mintz GS, Kimbiris D, Kalman P, et al. Two-dimensional echocardiographic determination of left atrial emptying volume: a non invasive index in quantifying the degree of nonrheumatic mitral regurgitation. J Am Coll Cardiol 1983;2:729-36.

11. Toutouzas K, Trikas A, Pitsavos C, Barbetseas J, Androulakis A, Stefanadis C, et al. Echocardiographic features of left atrium in elite male athletes. Am J Cardiol 1996;78:13147.12.Manning WJ, Silverman M, Katz SE, Douglas PS. Atrial ejection force: a noninvasive assessment of atrial systolic function. J Am Coll Cardiol 1993;22:221-5.

13. Mattioli AV, Castelli A, Andria A, Mattioli G. Clinical and echocardiographic features influencing recovery of atrial function after cardioversion of atrial fibrillation. Am J Cardiol 1998;82:1368-71.

14. Sparks PB, Jayaprakash S, Mond HG, Vohra JK, Grigg LE, Kalman JM. Left atrial mechanical function after brief duration atrial fibrillation. J Am Coll Cardiol 1999;33:342-9.

15. Manning WJ, Silverman DI, Katz SE, Riley MF, Come PC,
Doherty RM, et al. Temporal dependence of the return of atrial mechanical function on the mode of cardioversion of atrial fibrillation to sinus rhythm. Am J Cardiol 1995;75:624-6.

16. Thamilarasan M, Klein AL. Factors relating to left atrial enlargement in atrial fibrillation: "chicken or the egg" hypothesis. Am Heart J 1999;137:381-3.

17. Tsan TSM, Barnes ME, Bailey KR, Leibson CL, Montgomery SC, Takemoto Y, et al. Left atrial volume: important risk marker of incident atrial fibrillation in 1655 older men and women. Mayo Clin Proc 2001;76:467-75.

18. Keren G, Sherez J, Megidish R, Levitt B, Laniado S. Pulmonary venous flow pattern-its relationship to cardiac dynamics: a pulsed Doppler echocardiographic study. Circulation 1985; 71:1105-12.

19. Basnight MA, Gonzales MS, Kershenovich SC, Appleton CP. Pulmonary venous flow velocity: relation to hemodynamics, mitral floe velocity and left atrial volume, and ejection fraction. J Am Soc Echocardiogr 1991;4:547-58.

20. Goette A, Honeycutt C, Langberg JJ. Electrical remodeling in atrial fibrillation: time course and mechanisms. Circulation 1996;94:2968-74.

21. Allessie M, Konings K, Wijffels M. Electrophysiological mechanism of atrial fibrillation. In: DiMarco JP, Prystowsky JP, Holmes EN, editors. Atrial arrhythmias: state of the art. Armonk, NY: Futura Publishing Co; 1995. p. 155-61.

22. Braunwald E, Frahm CJ. Studies on Starling's law on the heart IV: observations on the hemodynamic functions of the left atrium in man. Circulation 1961;24:633-42.

23. Mihm MJ, Fushun Y, Carnes CA, Reisen PJ, McCarthy PM, Van Wagoner DR, et al. Impaired myofibrillar energetics and oxidative injury during human atrial fibrillation. Circulation 2001;104:174-80. 